\title{
Three-stage lattice relaxation of Ge islands on Sil111) measured by tunneling microscopy
}

\section{Citation}

Theiss, Silva K., D. M. Chen, and J. A. Golovchenko. 1995. Three-Stage Lattice Relaxation of Ge Islands on Si(111) Measured by Tunneling Microscopy. Applied Physics Letters 66, no. 4: 448. doi:10.1063/1.114052.

\section{Published Version}

doi:10.1063/1.114052

\section{Permanent link}

http://nrs.harvard.edu/urn-3:HUL.InstRepos:29406267

\section{Terms of Use}

This article was downloaded from Harvard University's DASH repository, and is made available under the terms and conditions applicable to Other Posted Material, as set forth at http:// nrs.harvard.edu/urn-3:HUL.InstRepos:dash.current.terms-of-use\#LAA

\section{Share Your Story}

The Harvard community has made this article openly available.

Please share how this access benefits you. Submit a story.

\section{Accessibility}




\title{
Three-stage lattice relaxation of $\mathrm{Ge}$ islands on $\mathrm{Si}(111)$ measured by tunneling microscopy
}

\author{
Silva K. Theiss \\ Department of Physics, Harvard University, Cambridge, Massachusetts 02138 \\ D. M. Chen \\ Rowland Institute for Science, Cambridge, Massachusetts 02142 \\ J. A. Golovchenko \\ Department of Physics and Division of Applied Sciences, Harvard University, Cambridge, Massachusetts \\ 02138 and Rowland Institute for Science, Cambridge, Massachusetts 02142
}

(Received 8 August 1994; accepted for publication 22 November 1994)

\begin{abstract}
We use the tunneling microscope to measure the surface lattice spacing of Ge islands grown on $\mathrm{Si}(111)$ as a function of their height. It changes in three stages: (I) (0-50 layers tall) Rapid relaxation from near the bulk $\mathrm{Si}$ value, at the end of which the lattice spacing atop some of the islands exceeds that of bulk Ge. (II) (50-80 layers) Rapid decrease in surface lattice spacing, to nearly $2 \%$ below the bulk Ge value. (III) (>80 layers) Gradual relaxation to the bulk value. Additional observations of dislocations and analysis of island widths are used to explain this behavior. (C) 1995 American Institute of Physics.
\end{abstract}

Strain relaxation in heteroepitaxial semiconductor films has recently been the subject of intense and fruitful study. A surprisingly rich and varied array of strain relaxation mechanisms, both elastic and plastic, has been observed. The important role of elastic relaxation in the early stages of heteroepitaxial growth has only lately been demonstrated: Recent transmission electron microscope (TEM) work has shown directly that islands can form prior to the introduction of any dislocations in both the Ge/Si system ${ }^{1,2}$ and the $\mathrm{In}_{x} \mathrm{Ga}_{1-x} \mathrm{As} / \mathrm{GaAs}$ system. ${ }^{3}$ Reflection high-energy electron diffraction (RHEED) studies of the $\operatorname{In}_{x} \mathrm{Ga}_{1-x} \mathrm{As} / \mathrm{GaAs}^{4-6}$ and $\mathrm{PbTe} / \mathrm{EuTe}^{7}$ systems also imply the presence of partially relaxed, coherent islands under certain growth conditions. Theoretical work has shown that coherent islands can be the equilibrium configuration under certain circumstances. ${ }^{8,9}$

The in-plane lattice constant of heteroepitaxial films as a function of coverage has been measured by several techniques. In studies of $\mathrm{Ge} / \mathrm{Si}(001)$ by $\mathrm{RHEED}^{10}$ and grazing incidence $\mathrm{x}$-ray diffraction, ${ }^{11}$ and RHEED studies of InAs/GaAs, ${ }^{4}$ lattice constants intermediate between those of the bulk substrate and the bulk overlayer were found after the onset of islands. However, previous scanning tunneling microscope (STM) measurements of Ge islands on both $\mathrm{Si}(001)^{12}$ and $\mathrm{Si}(111)^{13}$ suggested that the island tops were fully relaxed to the Ge lattice parameter, although lattice constant measurements were not a major focus of either study and in both cases the uncertainty was relatively large. Our preliminary STM results ${ }^{14}$ showed that surface lattice constants intermediate between those of $\mathrm{Si}$ and Ge could be measured, and hinted that they might overshoot that of bulk Ge at certain island heights.

In this work we demonstrate the application of the STM to the accurate measurement of the in-plane surface lattice spacing $a_{s}$ of a heteroepitaxial film. We study $a_{s}$ of individual Ge islands growing on $\mathrm{Si}(111)$ as a function of their height. Typically, the height of an island can be measured with an uncertainty of about $2 \%$, and $a_{s}$ can be measured with an uncertainty of a few tenths of a percent. Although we find, as expected, that $a_{s}$ initially increases rapidly with height and that it ultimately approaches the bulk Ge value, there are two surprises. First, near the end of the initial relaxation, around 40-60 monolayers height $(1 \mathrm{ML}=3.14 \AA$ high), $a_{s}$ for some of the islands clearly exceeds that of bulk Ge. Second, after the initial relaxation, there is a region where the magnitude of the strain in the top of the island dramatically increases again. In this region, $a_{s}$ decreases by about $2 \%$, dropping back well below the bulk Ge lattice constant.

The advantage of using the STM in this study is that $a_{s}$ can be measured on individual islands whose size and shape can be determined independent of any other features on the surface. There is no averaging over multiple islands that may not all be of the same size, as is necessarily the case with a diffraction technique. Additionally, no thinning of the sample, such as is required for TEM, is needed. The disadvantage is that the uncertainty is greater than that from a diffraction measurement. Furthermore, the procedure is tedious. It is necessary to search a large region of the sample to find islands in the height range of interest, and once such an island is found a large amount of data must be taken to obtain good statistics.

Experiments were performed with a home-built STM housed in a vacuum chamber with a base pressure of $6 \times 10^{-11}$ Torr. The chamber is equipped with a sputter gun, a low-energy electron diffraction/Auger electron spectroscopy system, a Ge effusion cell, and a load lock for sample exchange. We used As-doped, $0.01 \Omega \mathrm{cm}$ (111)-oriented Si substrates, 1 in. $\times 0.2$ in. $\times 0.02$ in. Clean $\mathrm{Si}$ surfaces were prepared by neon-ion sputtering, followed by annealing to $1250{ }^{\circ} \mathrm{C}$ for $30 \mathrm{~s}$. The procedure produces clean $\mathrm{Si}$ surfaces with about $1000 \AA$ wide terraces of the $7 \times 7$ reconstruction. Ge was deposited on one-half of the substrate for times ranging from 7.5 to $25 \mathrm{~min}$ at a rate of approximately $0.4 \mathrm{ML} /$ min, calibrated by Rutherford backscattering. The deposition 
temperature was $500{ }^{\circ} \mathrm{C}$ as measured by an optical pyrometer.

The clean Si half of the surface was used to calibrate the STM tip scanner for height, lattice constant, and area measurements on the Ge islands. Island heights were compared to that of $1 \mathrm{ML} \mathrm{Si}(111)$ steps. Since the thickness of the Ge wetting layer cannot be determined by STM, heights were measured above the lowest exposed level of the wetting layer, rather than above the $\mathrm{Ge} / \mathrm{Si}$ interface. Interatomic distances were calibrated against the spacing between the corner holes of the $7 \times 7$ reconstruction. Details of the measurement technique are given below. The width of an island was characterized by the square root of the area of its top facet. Area measurements were calibrated from the known area of $7 \times 7$ unit cells.

The sample preparation procedure typically produced triangular Ge islands that were widely spaced (several thousand $\AA$ apart) and greater than 100 ML tall. The wetting layer between the islands was rough on a scale of $2-3 \mathrm{ML}$ and consisted of Ge mesas, typically $200 \AA$ across and $600 \AA$ apart, as we have discussed previously. ${ }^{14}$

The details of the lattice measurement procedure are illustrated in Fig. 1. The top of a 54 ML high island is shown in Fig. 1(a). It has a mixture of the reconstructions found on bulk $\mathrm{Ge}(111): c(2 \times 8), c(2 \times 4)$, and $2 \times 2$, as marked. The tip height versus lateral position data shown in Fig. 1(b) were taken along the white line. Data were always taken along the fast scanning (horizontal) direction, or as close thereto as the crystal orientation allowed, to reduce the effect of drift. In this case the fit is simply a sine wave plus linear terms. Additional terms in the Fourier series are required to fit other reconstructions.

Figure 2 shows $a_{s}$ as a function of island height. Eightyfive percent of our data fell in the shaded region. The first two data points are the average of $a_{s}$ measured on several mesas that lay two or three layers, respectively, above the lowest exposed layer. These are part of the wetting layer, not truly islands, but are still found to have some of their strain relieved. Uniformly strained films can be unstable to the formation of small-amplitude oscillations which allow elastic relaxation $;{ }^{15}$ presumably the roughening is a manifestation of this effect.

On Ge islands with heights between about 2 and $40 \mathrm{ML}$ the STM reveals a continuously increasing level of relaxation. Remarkably, some of the islands 40-60 ML tall have a lattice spacing that actually exceeds that of bulk Ge(111) by around 1\%, as can be seen in Fig. 2. The reasons for this are not clear. However, in a finite-element calculation on the top surface of roughly cubic, coherent islands, the strain was found to become very weakly tensile. ${ }^{16}$ In this calculation the interface was constrained to stay flat. In very recent work based on linear elasticity in two-dimensional islands, it has been shown that if the interface under the islands is allowed to deform elastically, the lattice spacing on the top of the island can exceed the bulk Ge spacing by as much as $0.5 \%{ }^{17}$

At slightly greater heights, there are several islands that have a lattice spacing significantly below the bulk Ge lattice constant, and $a_{s}$ clearly goes through a minimum for islands near 70-80 ML in height. A similar dip in lattice constant
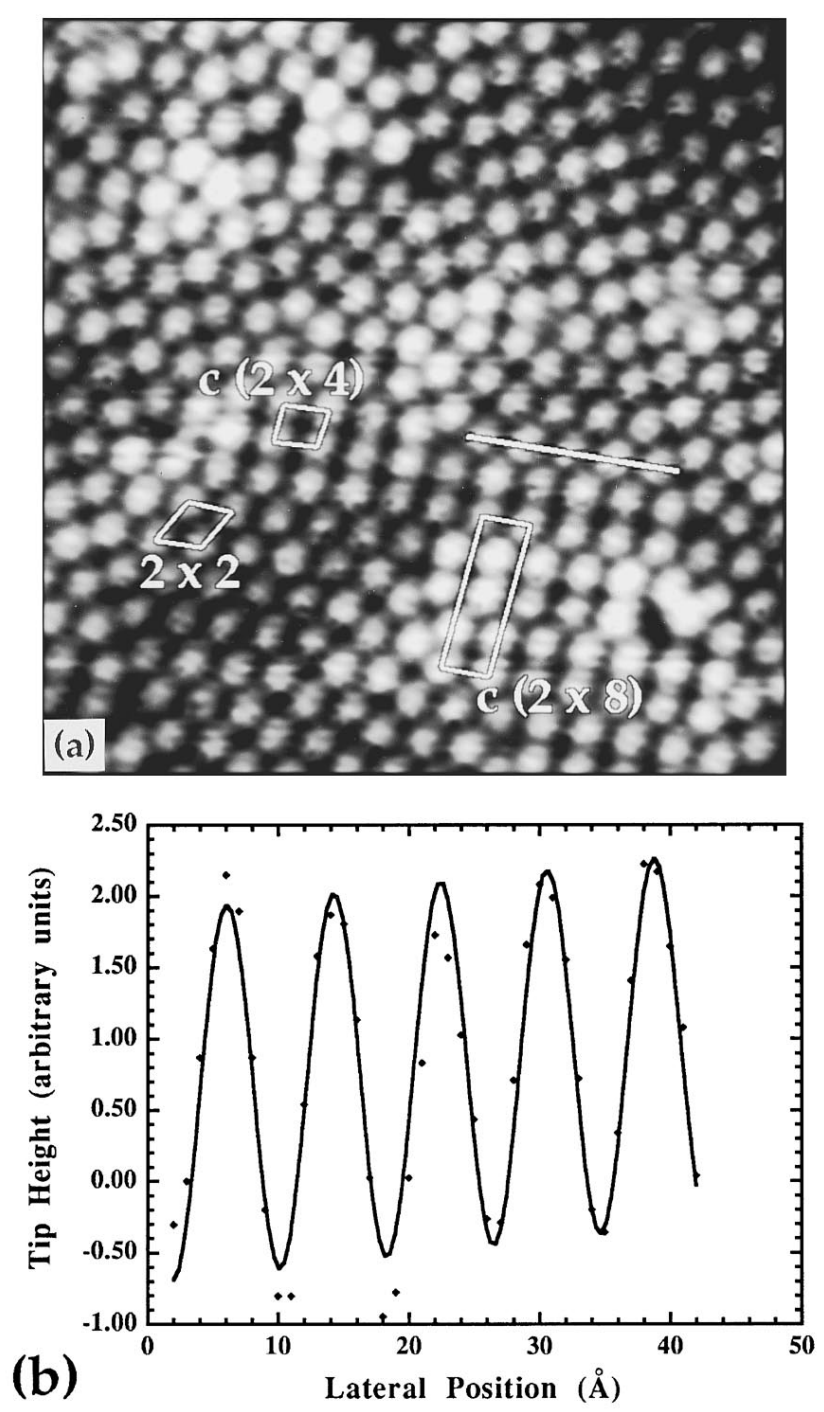

FIG. 1. (a) $140 \times 140 \AA$ scan of the top of a 54 ML high island, with $c(2 \times 8)$, $c(2 \times 4)$, and $2 \times 2$ reconstructions and a line cut marked. (b) Data from the line cut fit with a sine function.

has been seen under certain growth conditions in RHEED studies of $\operatorname{InAs} / \mathrm{GaAs}(001)^{4}$ and of $\mathrm{Ge} / \mathrm{Si}(001),{ }^{10}$ although the reasons for the dip were not discussed. We find that the decrease in $a_{s}$ is correlated with a change in the width of islands in this height range. The square root of the area of the top facet on the islands is plotted as a function of their height in Fig. 3. In the range from 0-50 ML the size of the top facet is nearly constant. Around $50 \mathrm{ML}$, however, there is a sharp increase in the area as a function of height. This corresponds to the region of decreasing $a_{s}$ on the relaxation graph. Since under our deposition conditions the islands are typically a few thousand $\AA$ apart, this is not due to the coalescence of islands.

Above about $80 \mathrm{ML}, a_{s}$ again increases with island height. Strain-relieving defects that penetrate the top surface of the island are observed in $64 \%$ of these islands, ${ }^{18}$ while they are found in only $14 \%$ of the islands shorter than 80 ML. In this region the island width increases gradually.

We propose the following model for the three stages of growth. In stage I the islands gain height rapidly. This leads 


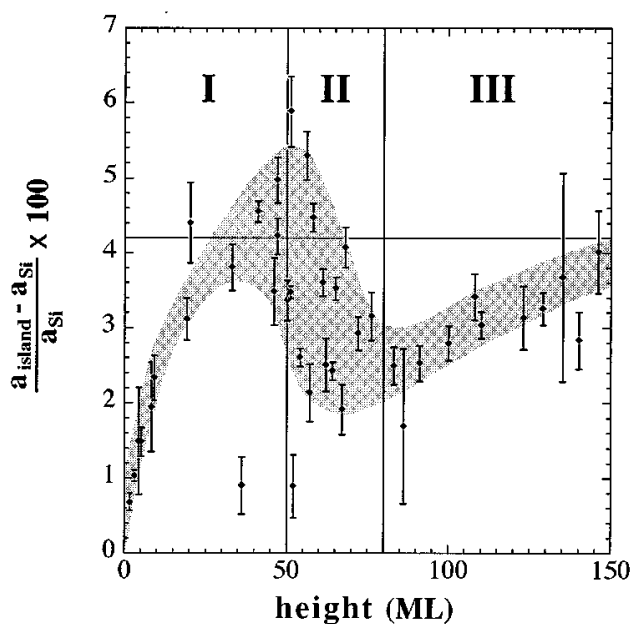

FIG. 2. Lattice parameter $a_{s}$ of Ge islands vs island height. $a_{s}$ is expressed as a percentage difference from the bulk Si lattice constant. Vertical lines roughly divide the three different stages of growth. The horizontal line corresponds to $a_{s}$ for bulk Ge.

to the greatest possible height-to-width aspect ratio, which allows the greatest degree of elastic relaxation. ${ }^{8,16}$ The substrate participates in the relaxation, which can lead to $a_{s}$ on some of the islands exceeding that of bulk Ge. ${ }^{17}$ Around 40-50 ML, it becomes unfavorable for the islands to continue to increase in height, although the nature of the limita-

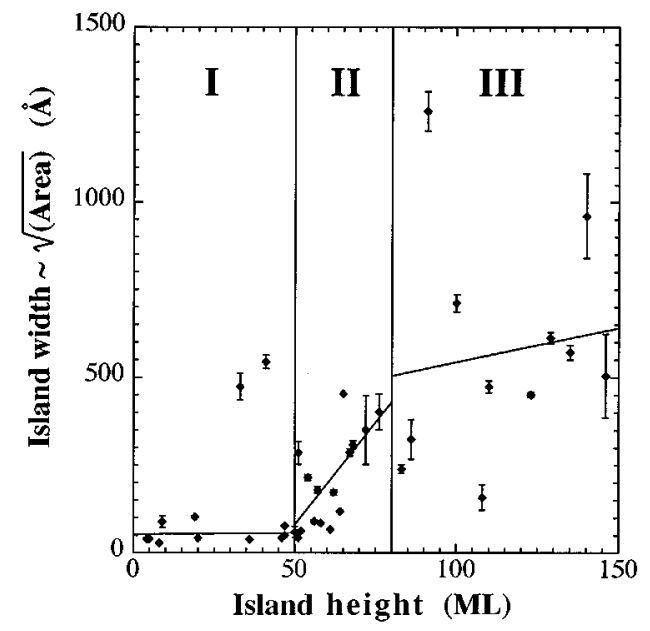

FIG. 3. Approximate width of the islands expressed as $\sqrt{ }$ (area of top facet) vs island height. Vertical lines correspond to those in Fig. 2. Line fit in the first region omits the two outlying points. Line fits in second and third regions are to all points. tion is not presently clear. In stage II, further coherent growth takes place by an increase in the width of the islands. However, this diminishes their ability to relieve strain elastically, and they forfeit some of the relaxation they had achieved. The width of the shaded region in Fig. 2 suggests that the details of this stage of growth may be a sensitive function of deposition conditions. Inaccuracies in the sample temperature measurement, as well as variations in the temperature along the sample, may cause the observed range of properties. The increasing strain produced by the widening of coherent islands rapidly becomes unfavorable also, and so around $80 \mathrm{ML}$ dislocations are introduced. Strain relaxation in stage III proceeds by dislocation introduction. This is not a very efficient process, and the islands relax slowly to their bulk lattice constant with increasing size.

We hope that the new, local approach to this problem provided by the tunneling microscope will stimulate further study of the processes involved in heteroepitaxy.

The authors thank F. Spaepen and C. W. Snyder for helpful discussions. Supported by JSEP (N00014-89-J-1023) and the MRL at Harvard (NSF-DMR-8920490).

${ }^{1}$ D. J. Eaglesham and M. Cerullo, Phys. Rev. Lett. 64, 1943 (1990).

${ }^{2}$ F. K. LeGoues, M. Copel, and R. M. Tromp, Phys. Rev. B 42, 11690 (1990).

${ }^{3}$ S. Guha, A. Madhukar, and K. C. Rajkumar, Appl. Phys. Lett. 57, 2110 (1990).

${ }^{4}$ H. Munekata, L. L. Chang, S. C. Woronick, and Y. H. Kao, J. Cryst. Growth 81, 237 (1987).

${ }^{5}$ C. W. Snyder, B. G. Orr, D. Kessler, and L. M. Sander, Phys. Rev. Lett. 66, 3032 (1991).

${ }^{6}$ J. Massies and N. Grandjean, Phys. Rev. Lett. 71, 1411 (1993).

${ }^{7}$ G. Springholz and G. Bauer, Phys. Rev. B 48, 10998 (1993).

${ }^{8}$ C. Ratsch and A. Zangwill, Surf. Sci. 293, 123 (1993).

${ }^{9}$ D. Vanderbilt and L. K. Wickham, Mater. Res. Soc. Symp. Proc. 202, 555 (1991).

${ }^{10}$ T. Sakamoto, K. Sakamoto, K. Miki, H. Okumura, S. Yoshida, and H. Tokumoto, NATO ASI Series B 239, 263 (1990).

${ }^{11}$ A. A. Williams, J. M. C. Thornton, J. E. Macdonald, R. G. van Sifhout, J. F. van der Veen, M. S. Finney, A. D. Johnson, and C. Norris, Phys. Rev. B 43, 5001 (1991).

${ }^{12}$ Y.-W. Mo, D. E. Savage, B. S. Swartzentruber, and M. G. Lagally, Phys. Rev. Lett. 65, 1020 (1990).

${ }^{13}$ U. Köhler, O. Jusko, G. Pietsch, B. Müller, and M. Henzler, Surf. Sci. 248, 321 (1991).

${ }^{14}$ Silva K. Theiss, D. M. Chen, and J. A. Golovchenko, Mater. Res. Soc. Symp. Proc. 317, 15 (1994).

${ }^{15}$ M. Grinfeld, Europhys. Lett. 22, 723 (1993); B. J. Spencer, P. W. Voorhees, and S. H. Davis, J. Appl. Phys. 73, 4955 (1993); Phys. Rev. Lett. 67, 3696 (1991); D. J. Srolovitz, Acta Metall. 37, 621 (1989).

${ }^{16} \mathrm{P}$. Van Mieghem, S. C. Jain, J. Nijs, and R. Van Overstraeten, J. Appl. Phys. 75, 666 (1994).

${ }^{17}$ L. J. Gray, M. F. Chisholm, and T. Kaplan, in Boundary Element Technology VIII, edited by H. Pina and C. A. Brebbia (Southampton, Boston, MA 1993), pp. 181-190; private communication.

${ }^{18}$ Only dislocations that penetrate the top surface of the islands can be observed. Dislocations that just penetrate the sides of the islands cannot be detected. 